\title{
Yoga and Cardiovascular Health Trial (YACHT): a UK-based randomized mechanistic study of a yoga intervention plus usual care versus usual care alone following an acute coronary event, BMJ Open 2019;9:e030119
}

\author{
Saras Jyoti \\ Amity Institute of Molecular Medicine and Stem Cell Research, Amity University, NOIDA, India
}

\author{
Saras Jyoti \\ Amity Institute of molecular medicine and stem cell research \\ Amity University \\ NOIDA, India \\ Contact no: +91-9816773821 \\ E-mail: saras.jyoti@gmail.com
}

\section{Introduction}

Over the years Yoga has gained popularity throughout the world because of its various health benefits. Yoga is mindbody intervention which includes breathing, body postures and meditation (1). The studies on Yoga have documented to lower the stress and depression and are believed to improve biological cardiovascular risk factors (2-4). Cardiac rehabilitation (CR) is a program designed to strengthen cardiovascular health if you have experienced the heart related problems, and it has been shown to improve cardiovascular mortality and hospital re-admissions in patients with coronary heart disease (CHD). Yoga could, therefore, be a useful addition to CR. In this UK-based randomized report (Yoga and Cardiovascular Health Trial (YACHT), the hypothesis was that Yoga could be related to primarily with improvements in cardiovascular function in patients suitable for CR.

\section{Study Design}

For understanding the effects of Yoga practice on subclinical cardiovascular events, risk factors and neuro-endocrine pathways in patients receiving CR after acute coronary experiences. This was a 3-month study, two-arm (yoga+usual care vs usual care alone) parallel randomized mechanistic study. Participants' inclusion criteria was the referral to CR programme after an acute coronary syndrome (MI, PCI, coronary artery bypass grafting). Pre-specified inclusion criteria were age between of 35 and 80 years, male or female, without co morbid disease or mobility limitations that would preclude participation in CR. Overall 80 participants, were (68\% men, $60 \%$ South Asian) referred to CR programme in 2012-2014. Statistical analyses were performed using STATA V.15 software.

\section{Methods}

A certified Yoga instructor supervised Yoga classes which incorporated exercises such as stretching, breathing, healing imagery and deep relaxation. For inclusion analysis minimum 18 Yoga classes were necessary. The yoga intervention was given twice a week, group session basis for 12 weeks alongside the usual care, 6- to 12-week programme designed according to patient requirements which includes (a) ongoing risk factor monitoring/advice/support, (b) exercise sessions in a gym, led by cardiac physiologist or a home-based exercise programme, (c) health education lectures (led by CR sister, pharmacist, dietitian, clinical psychologist, cardiac physiologist), (d) relaxation sessions, (e) guidance and supervised use of the 'Edinburgh Heart Manual' (http://www.theheartmanual.com/Pages/default.aspx, accessed 26/9/2017). There were total 24 yoga classes. Main follow-up measures (i) Evaluated left ventricular filling pressure (E/e'), (ii) distance walked, fatigue and breathlessness in a 6 min walk test, (iii) blood pressure, heart rate and estimated peak VO2 following a $3 \mathrm{~min}$ step-test. The outcomes on hypothalamus-pituitary-adrenal axis, autonomic function, body fat, blood lipids and glucose, stress and general health were also analyzed.

\section{Implications}

Only 25 participants in the yoga + usual care group and 35 participants in the usual care group completed the study. After the 3-month intervention, E/e improved in both groups, but there was no evidence of yoga-related additional benefit in diastolic function. In 6 Min walk test and 3 Min step-test, no evidence of yoga-related additional benefit found in participants. No other improvements in exercise testing and the other secondary outcomes like vascular measures, carotid IMP, HPA axis, autonomic function and metabolic measures were observed in the participants. It can be concluded that in this UK-based randomized mechanistic study, with 60 copmleting participants, there was no marked improvement related with the addition of a structured 3-month Yoga intervention to usual CR care in key cardiovascular and neuroendocrine outcomes shown to be responsive to yoga in previous mechanistic studies. 


\section{Inference}

- The study for the first time has shown to adopt a comprehensive approach for measuring cardiovascular clinical and subclinical effects of Yoga (plus usual cardiac rehabilitation (CR)) versus usual CR).

- Number of dropout in the yoga arm of the study exceeded that in the usual care arm (25 and 35 completed the study, respectively), which might be possible because of the burden of attending both Yoga training and usual CR.

- For some outcomes 3 months is a short time to obtain substantial regression.

- The study reports the real world setting for older people following an acute coronary event.
Received Date: 02-06-20; Revised Date: 08-08-20

Accepted Date: $11-08-20$

\section{References}

1. Feuerstein G, Wilber K. The Yoga Tradition: Its History, Literature, Philosophy and Practice. Prescott, Ariz: Hohm Press, 2008.

2. Choices N. A guide to yoga: department of health and social care, UK government. Available: https://www.nhs.uk/live-well/exercise/guide

3. Chong CSM, Tsunaka M, Tsang HWH, et al. Effects of yoga on stress management in healthy adults: a systematic review. Altern Ther Health Med 2011;17:32-8.

4. Cramer H, Lauche R, Langhorst J, et al. Yoga for depression: a systematic review and meta-analysis. Depress Anxiety 2013;30:1068-83.

doi: 10.38205/imcr.010245 